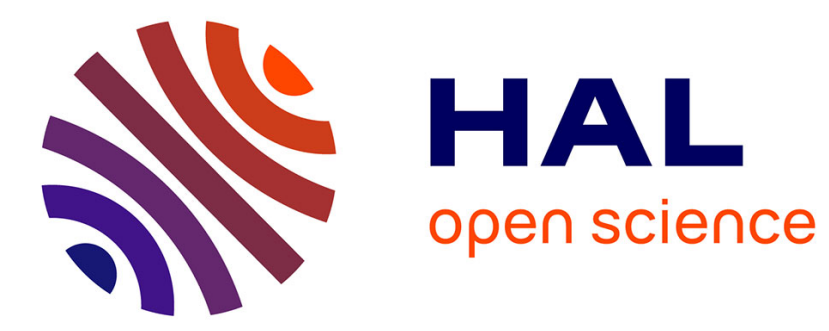

\title{
Agrafes à double crochet gallo-romaines trouvées en Normandie
}

\author{
Claude Jigan, Jean-Yves Marin
}

\section{To cite this version:}

Claude Jigan, Jean-Yves Marin. Agrafes à double crochet gallo-romaines trouvées en Normandie. Gallia - Fouilles et monuments archéologiques en France métropolitaine, 1984, 42 (2), pp.243-246. 10.3406/galia.1984.1921 . hal-01941044

\section{HAL Id: hal-01941044 \\ https://hal.science/hal-01941044}

Submitted on 27 Feb 2020

HAL is a multi-disciplinary open access archive for the deposit and dissemination of scientific research documents, whether they are published or not. The documents may come from teaching and research institutions in France or abroad, or from public or private research centers.
L'archive ouverte pluridisciplinaire HAL, est destinée au dépôt et à la diffusion de documents scientifiques de niveau recherche, publiés ou non, émanant des établissements d'enseignement et de recherche français ou étrangers, des laboratoires publics ou privés.

\section{(이) $\$$}

Distributed under a Creative Commons Attribution - NonCommercial - NoDerivatives| 4.0 


\title{
NOTE
}

\section{AGRAFES A DOUBLE CROCHET GALLO-ROMAINES TROUVÉES EN NORMANDIE}

\author{
par Claude JIGAN ef Jean-Yves MARIN
}

Si les agrafes à double crochet sont bien connues en ce qui concerne la période mérovingienne, période pour laquelle elles font l'objet de publications régulières ${ }^{1}$, il n'en est pas de même pour l'époque gallo-romaine. On a en effet considéré pendant de longues années ce modeste accessoire vestimentaire comme caractéristique $d u v_{\text {III }} \mathrm{e} s$. Le problème restant alors à résoudre, était de déterminer si ce type d'agrafe était uniquement utilisé pour fixer le suaire sur la poitrine du mort ${ }^{2}$ ou bien s'il était également employé par les vivants comme fermeture de vêtement ${ }^{3}$.

En 1963, M. Georges Fouet remettait radicalement en cause leur datation en annonçant la découverte de ces agrafes dans un contexte nettement caractérisé du milieu du Ive s. ${ }^{4}$. L'auteur concluait à une grande

1 W. Hubener, Merowingerzeilliche Kettenschmuckträger in Wesleuropa, Madrider Milteilungen, 12, 1971, pp. 241-261.

2 A. Audin, Destination des agrafes mérovingiennes à double crochel, dans Revue Archéologique de l'Est et du Centre-Est, 1955, t. VI, fasc. 2, pp. 158-159.

3 A. Roes, Agrafes du Haul Moyen-Age à double crochet, dans Revue Archéologique de l'Est et du Centre$E s t, 1954$, t. V, fasc. 4, pp. 330-334. - . Rilliot, Contribulion à l'élude des agrafes à double crochet, dans Revue Archéologique de l'Esl et du Centre-Est, 1966, t. XVII, fasc. 3-4, pp. 240-250.

4 G. Foure, Agrafes à double crochel du $I V^{\mathrm{e}}$ sieccle dans la villa de Monlmaurin (Haute-Garonne), dans ancienneté de ce mode d'attache qu'il pensait pouvoir faire remonter à la période de la T'ène. En $1972^{5}$, le même auteur mentionne de nouvelles agrafes également datées du $\mathrm{IV}^{\mathrm{e}} \mathrm{s}$. et provenant d'un site proche de celui où avait été effectuée la première découverte. Depuis lors, hormis quelques rares indications peu explicites, les agrafes décrites dans les publications ne concernent, à notre connaissance, que l'époque mérovingienne (habitats et nécropoles).

Or, depuis 1974, quatre sites de Normandie ont fourni des agrafes à double crochet dans des niveaux gallo-romains parfaitement scellés. Trois de ces sites sont situés dans le département du Galvados (Caen, Saint-Contest, Vieux), et le quatrième en Seine-Maritime (Rouen) ${ }^{6}$. Dans deux de ces cas, Gaen et Rouen, il s'agit de villes où l'épaisseur des dépôts, souvent très importante, exclut d'éventuelles infiltrations dans les niveaux les plus anciens,

Cellicum VI, 1963, Supplément à Ogam, no 82, pp. 277292.

5 G. Fouet, Le sanctuaire des eaux de "La Hillère" $\grave{a}$ Montmaurin, dans Gallia, 1972, t. 30, fasc. 1, pp. 111114.

6 Nous tenons à remercier Dominique et Patrick Halbout respectivement responsables des chantiers de Vieux et de Rouen, d'avoir bien voulu nous communiquer leurs agrafes à double crochet ainsi que toutes les données permettant leur datation. 


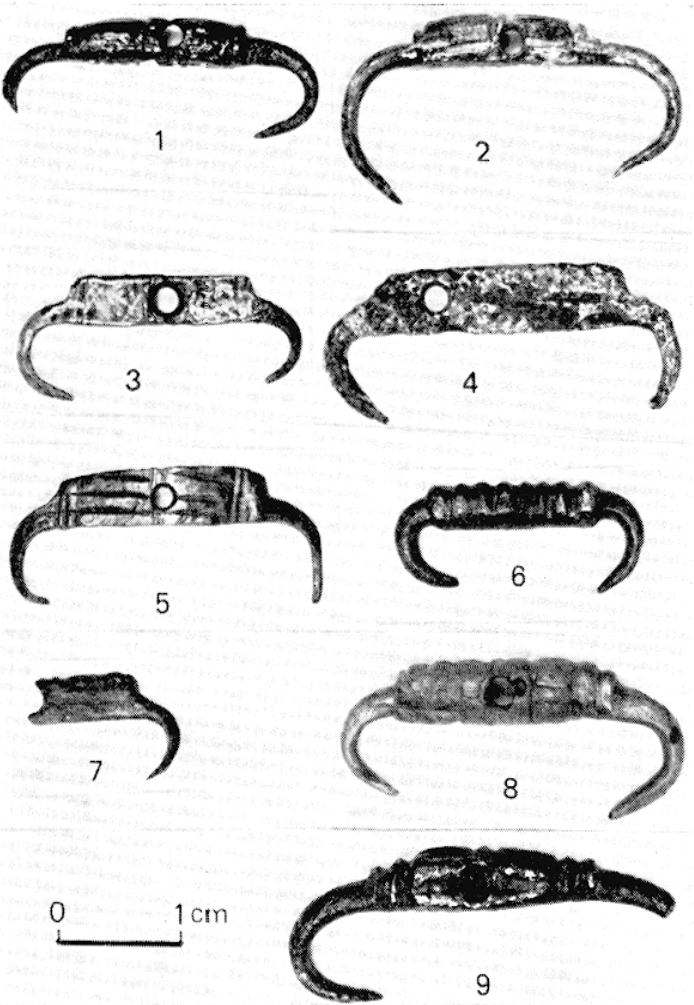

qui seraient immédiatement décelables au décapage. Le site de Saint-Contest el la parcelle de Vieux où furent découvertes des agrafes sont abandonnés dès l'antiquité et les agrafes sont en relation avec des niveaux d'occupation des $\mathrm{I}^{\mathrm{er}}$ et $\mathrm{II}^{\mathrm{e}} \mathrm{s}$. G'est pourquoi les datations que nous proposons ci-après nous semblent extrêmement fiables dans la quasitotalité des cas (seul cas douteux, celui de Caen $N^{\circ} 4$, où l'agrafe provient d'un remblai contenant du matériel du irI ${ }^{\mathrm{e}} \mathrm{s}$.).

Caen. - 1. Site de l'Ancienne École Normale d'Institutrices (fig. 1, 1). Datation $2^{e}$ moitié du $\mathrm{I}^{\mathrm{er}} \mathrm{s}$.

Long. : $25 \mathrm{~mm}$, diam. : $4 \mathrm{~mm}$.

L'agrafe est constituée d'un corps cylindrique de section circulaire prolongé aux extrémités par deux crochets dont un incomplet. De part et d'autre d'un annelet central, deux bourrelets sont limités à leur extrémité par un annelet. Chaque bourrelet est décoré de huit stries longitudinales. Un trou est percé dans le corps de l'annelet central.

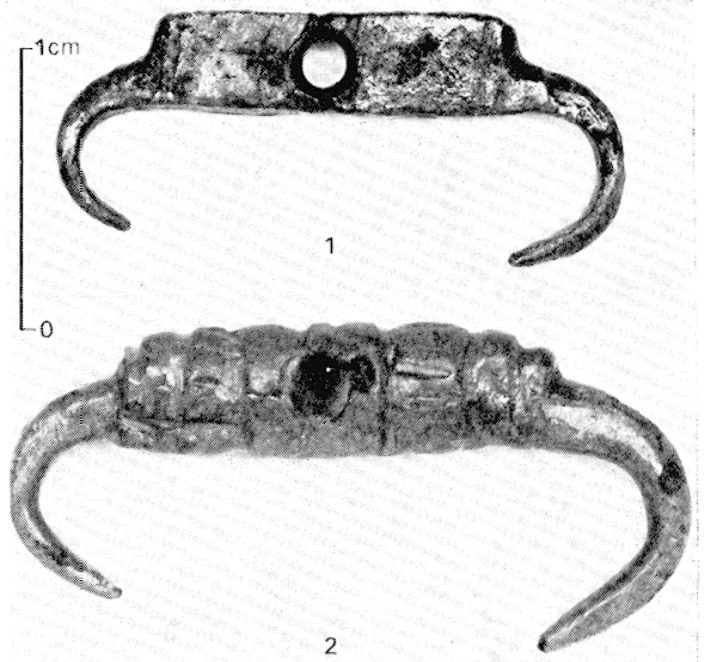

2 Agrandissement de 2 des agrales les plus significatives: Caen 3 et Saint-Contest 1.

1 Agrafes à double crochel gallo-romaines trouvées en Normandie : 1, 2, 3, 4 : Caen; 5,6 : Rouen; 7, 8 : Saint-Contest; $9:$ Vicux.

2. Site de la Salle des Gardes II (fig. 1,2). Datation : II $^{\mathrm{e}} \mathrm{s}$.

Long. : $27 \mathrm{~mm}$, diam. : $4 \mathrm{~mm}$.

Corps cylindrique de section circulaire prolongé aux extrémités par deux crochets inégaux. De part et d'autre d'un annelet central, deux bourrelets sont limités à leur extrémité par un annelet. Chaque bourrelet est décoré de quatre stries longitudinales dont deux sur la partie supérieure. Un trou est percé dans le corps de l'annelet central.

3. Site de la Salle des Gardes II (fig. 1,3; fig. 2, 1). Dalalion : fin du $\mathrm{II}^{\mathrm{c}} \mathrm{s}$.

Long. : $24 \mathrm{~mm}$, épais. : $2 \mathrm{~mm}$.

Corps en forme de parallélépipède rectangle très plat prolongé à chaque extrémité par deux crochets inégaux. Chaque face latérale est décorée au départ des crochets par deux stries parallèles. La face supérieure du parallélépipède est décorée de stries parallèles très serrées. Le corps est percé d'un trou central.

4. Site de la Mairie Provisoire (fig. 1,4). Datation : III $^{\mathrm{e}}$ s.? 
Long. : $28 \mathrm{~mm}$, épais. : $2 \mathrm{~mm}$. Mauvais état de conservation.

Corps en forme de parallélépipède rectangle, très plat, prolongé à chaque extrémité par deux crochets égaux. Ghaque face latérale est décorée par une gorge longitudinale. La face supérieure du parallélépipède est peut-être décorée de stries. Le corps est percé d'un trou nettement décentré.

Rouen. - 1. Site de La Haule-Vieille Tour (fig. 1,5). Datation : Iv $\mathrm{s}$.

Long. : $25 \mathrm{~mm}$, épais. : $2 \mathrm{~mm}$.

Corps en forme de parallélépipède rectangle très plat prolongé à chaque extrémité par deux crochets dont un incomplet. Chaque face latérale est décorée de stries longitudinales limitées au départ des crochets par des stries latérales. La face supérieure du parallélépipède est décorée de stries parallèles. Le corps est percé d'un trou central.

2. Site de La Haule-Vieille Tour (fig. 1, 6). Datation : ive $\mathrm{s}$.

Long. : $20 \mathrm{~mm}$, diam. : $3, \overline{5} \mathrm{~mm}$.

Corps cylindrique de section circulaire prolongé aux extrémités par deux crochets égaux. Composé de huit annelets identiques, le corps n'est pas percé.

Sannt-Gontest. - 1. Site du Cilos de Bilól (fig. 1,7). Datation: $\mathrm{I}^{\mathrm{er}} \mathrm{s}$.

Long. : $12 \mathrm{~mm}$, épais. : $2 \mathrm{~mm}$. Une demiagrafe conservée.

Corps en forme de parallélépipède rectangle très plat, prolongé à l'extrémité conservée par un crochet. Chaque face latérale est décorée d'une gorge longitudinale. La face supérieure du parallélépipède est décorée de deux rainures parallèles dont une est située au-dessus du trou central.

2. Site du Clos de Bilôl (fig. 1, 7; figr. 2, 2). Datation : $\mathrm{I}^{\mathrm{er}} \mathrm{s}$.

Long. : $27 \mathrm{~mm}$, diam. : $\overline{\mathrm{j}} \mathrm{mm}$.

Corps cylindrique de section circulaire prolongé aux extrémités par deux crochets inégaux. De part et d'autre d'un bourrelet médian percé d'un trou, deux petites gorges séparent deux annelets successifs. Ils sont décorés de stries longitudinales.

VIEux. - Parcelle AH 21 cadastre 1964 (fig. 1,8). Datation : $\mathrm{II}^{\mathrm{e}} \mathrm{s}$.

I song. : $25 \mathrm{~mm}$, diam. : $5 \mathrm{~mm}$.

Corps cylindrique de section circulaire prolongé aux extrémités par deux crochets dont un incomplet. De part et d'autre d'un annelet médian percé d'un trou, deux bourrelets sont décorés de stries longitudinales. Ils sont limités aux extrémités par deux annelets.

$*$

Dans la mesure où aucune de ces agrafes ne provient de fouilles de nécropoles, nous ne possédons pas d'éléments nouveaux concernant leur fonction. C'est pourquoi nous nous rangeons volontiers à l'opinion de G. Fouet : :Selon le cas, épingle de sûreté, attache ou maillon mobile, sommaire fibule, cette agrafe polyvalente devait rendre bien des services dans la vie quotidienne des humbles avant d'être parfois sacrifiée pour fermer les linceuls $\rangle^{7}$.

L'étude de ces neuf agrafes permet d'avancer certaines constatations si on les compare avec celles du vil ${ }^{e}$ s. trouvées dans des nécropoles de Normandie ${ }^{8}$.

Toutes les agrafes que nous venons de décrire sont en bronze. $A$ une exception près (Rouen 2), elles sont perforées d'un trou d'un diamètre de l'ordre du millimètre. Sur ces deux premières caractéristiques aucune différence notable avec les agrafes de l'époque mérovingienne n'est à relever. Parmi ces dernières, une

7 G. Focle, 1963, op. cil., p. 280.

8 Ces publications sont nombreuses, nous ne citerons que les principales : M. de BoüarD-G. MASt, Un nouveau cimelière du Haul Moyen-Age à Fleury-surOrne (Calvados), dans Annales de Normandie, juin 1964, pp. 111-131. - C. Pir.et, La nécropole de Frénouville, dans BAR Inlernalional Série 8.3, 1980, I. I, pp. 79-80. - J. levalet-J. Lemisk:, Sainl-Marlin de Verson (Galvados), nécropole des $V I I^{\mathrm{e}}$ et $V I I I^{\mathrm{e}}$ siècles, dans Archéologie Médiévale, 1980, t. X, pp. 59-104. C. Lonren, Le Village de Saint-Martin de Mondeville (Calvados). Premiers résultats de fouilles (1978-1980), dans Westvlaamse Archaeologica Minografieën II, De Merovingische beschaving in de Scheldevallei, Kortrijk, 1981 , pp. 169-198. 
seule est en fer et non percée 9 . Il arrive, comme c'est le cas pour l'une des nôtres (Gaen 4), que le trou soit nettement décentré.

La forme générale est constante ; le corps est soit de section circulaire, soit en forme de parallélépipède rectangle très aplati. Par contre, les agrafes filiformes et les agrafes à crochets repliés sous une face ne sont pas représentées en Normandie.

C'est sur la forme du corps qu'il nous parait exister une différence sensible entre les deux périodes; en effet, si à l'époque gallo-romaine les corps cylindriques de section circulaire coexistent avec ceux en forme de parallélépipède rectangle, seule la seconde forme est représentée dans les sites mérovingiens fouillés jusqu'à maintenant. Ce pourrait être là un élément de dalation assez précis si cela devait se confirmer dans d'autres régions.

9 C. Lorren, op. cit., p. 184.
On peut aussi noter qu'à l'exception de Rouen 2, les agrafes à corps cylindrique présentent une certaine analogie dans le décor (Gaen 1-2, Saint-Contest 2, Vieux).

Les dernières remarques s'appliquent aussi bien à l'époque gallo-romaine qu'à l'époque mérovingienne : ainsi, il semble que toutes les agrafes soient moulées, puis un décor incisé a été ajouté, avec plus ou moins de bonheur. La quasi-totalité des agrafes sont d'une longueur oscillant entre 20 et 30 millimètres. Enfin, on rencontre simultanément des agrafes rudimentaires à peine décorées et d'autres de belle facture.

Il est sans doute trop tôt pour tirer des conclusions générales ; cependant il nous a paru intéressant de faire connaître ces résultats dès maintenant. Seule une enquête menée à plus grande échelle permettrait d'affiner la typologie de cet accessoire, le faisant ainsi devenir un élément de datation plus utilisable.

Claude JiGAN et Jean-Yves MARIN 